Research Article

\title{
On the vertex-degree based invariants of digraphs
}

\author{
Hanyuan Deng, Zikai Tang*, Jiaxiang Yang, Jing Yang, Meiling You
}

Key Laboratory of Computing and Stochastic Mathematics (Ministry of Education), College of Mathematics and Statistics, Hunan Normal University, Changsha, Hunan 410081, P. R. China

(Received: 6 September 2021. Accepted: 20 September 2021. Published online: 27 September 2021.)

(C) 2021 the authors. This is an open access article under the CC BY (International 4.0) license (www.creativecommons.org/licenses/by/4.0/).

\begin{abstract}
Let $D=(V, A)$ be a digraph without isolated vertices. A vertex-degree based invariant $I(D)$ related to a real function $\varphi$ of $D$ is defined as $I(D)=\frac{1}{2} \sum_{u v \in A} \varphi\left(d_{u}^{+}, d_{v}^{-}\right)$, where $d_{u}^{+}$(respectively, $d_{u}^{-}$) denotes the out-degree (respectively, in-degree) of a vertex $u$. In this paper, we give the extremal values and extremal digraphs of $I(D)$ over all digraphs with $n$ non-isolated vertices. By applying the obtained results, we determine the extremal values of some well-known vertexdegree based topological indices of digraphs, such as the Randić index, the Zagreb indices, the sum-connectivity index, the geometric-arithmetic index, the atom-bond connectivity index and the harmonic index, and characterize the corresponding extremal digraphs.
\end{abstract}

Keywords: graph invariant; digraph; Randić index; Zagreb indices; sum-connectivity index; geometric-arithmetic index; atom-bond connectivity index; harmonic index.

2020 Mathematics Subject Classification: 05C20, 05C35, 05C07, 05 C92.

\section{Introduction}

A digraph $D=(V, A)$ is an ordered pair $(V, A)$ consisting of a non-empty finite set $V$ of vertices and a finite set $A$ of ordered pairs of distinct vertices called arcs (in particular, $D$ has no loops). If $a \in A$ is an arc from vertex $u$ to vertex $v$, then we indicate this by writing $a=u v$. The vertex $u$ is the tail of $a$ and the vertex $v$ is its head. The out-degree (respectively, in-degree) of a vertex $u$, denoted by $d_{u}^{+}$(respectively, $d_{u}^{-}$) is the number of arcs with tail $u$ (respectively, with head $u$ ). A vertex $u$ for which $d_{u}^{+}=d_{u}^{-}=0$ is called an isolated vertex. We denote by $\mathcal{D}_{n}$ the set of all digraphs with $n$ non-isolated vertices.

Recently, J. Monsalve and J. Rada [7] extended the concept of vertex-degree based topological indices of graphs to digraphs. They obtained the extremal values of the Randic index of digraphs over $\mathcal{D}_{n}$, and found the extremal values of the Randić index over the set of all oriented trees with $n$ vertices. Also, they found the extremal values of the Randić index over the set of all orientations of the path, the cycle with $n$ vertices and the hypercube $H_{d}$ of dimension $d$, respectively.

All the digraphs considered in this paper are strict, i.e., no loops and no two arcs with the same ends have the same orientation.

A vertex-degree-based (VDB, for short) VDB invariant (or VDB topological index) $I(D)$ related to a real function $\varphi$ of a digraph $D$ with $n$ non-isolated vertices is defined as

$$
I(D)=\frac{1}{2} \sum_{1 \leq i, j \leq n-1} a_{i j} \varphi_{i j}
$$

where $\varphi_{i j}=\varphi(i, j)$ and $a_{i j}$ is the number of $\operatorname{arcs}$ in $D$ of the form $u v$ such that $d_{u}^{+}=i$ and $d_{v}^{-}=j$, i.e., $(i, j)$-arcs in $D$.

Recall that if $G$ is a graph, we can identify $G$ with the symmetric digraph $\vec{G}$ by replacing every edge of $G$ with a pair of symmetric arcs. Under this correspondence,

$$
I(G)=\sum_{1 \leq i \leq j \leq n-1} m_{i j} \varphi_{i j}=I(\vec{G})
$$

for any VDB topological index $\varphi$ with $\varphi_{i j}=\varphi_{j i}$ (symmetric) and $m_{i j}$ the number of edges in $G$ joining vertices of degree $i$ and $j$. In other words, The VDB topological index of digraphs is a generalization of the concept of VDB topological index of graphs. 
In fact, a VDB topological index $I(D)$ of a digraph is an invariant based on the weights of all arcs depending on the out degrees of their tails and the in-degrees of their heads, i.e.,

$$
I(G)=\sum_{u v \in A} \varphi\left(d_{u}^{+}, d_{v}^{-}\right)
$$

where $\varphi(x, y)$ is a real function of $x$ and $y$ with $\varphi(x, y) \geq 0$ and $\varphi(x, y)=\varphi(y, x)$.

(i). If $\varphi(x, y)=(x y)^{\alpha}$, where $\alpha \neq 0$ is a real number, then $I(D)$ is the general Randić index of a digraph $D$. Furthermore, $I(D)$ is the Randić index, the second Zagreb index and the second modified Zagreb index for $\alpha=-\frac{1}{2}, \alpha=1$ and $\alpha=-1$, respectively. For these indices of graphs, see [1,6, 8,9].

(ii). If $\varphi(x, y)=(x+y)^{\alpha}$, then $I(D)$ is the general sum-connectivity index of a digraph $D$. Further, $I(G)$ is the sumconnectivity index and the first Zagreb index for $\alpha=-\frac{1}{2}$ and $\alpha=1$, respectively. See $[5,8,11,12]$ for graphs.

(iii). If $\varphi(x, y)=\frac{\sqrt{x y}}{\frac{1}{2}(x+y)}$, then $I(D)$ is the first geometric-arithmetic index $G A$ of a digraph $D$. See [10] for the first geometric-arithmetic index of a graph.

(iv). If $\varphi(x, y)=\sqrt{\frac{x+y-2}{x y}}$, then $I(G)$ is the atom-bond connectivity $(A B C)$ index of a digraph $D$. See [3] for the atom-bond connectivity index of a graph.

(v). If $\varphi(x, y)=\frac{2}{x+y}$, then $I(D)$ is the harmonic index of a digraph $D$. See [4] for the harmonic index of a graph.

In this paper, we give the extremal values and extremal graphs of the VDB topological indices over all digraphs with $n$ non-isolated vertices by a unified linear-programming modeling, and provide a unified approach to determining some extremal values and characterizing extremal digraphs of Randić index, Zagreb indices, sum-connectivity index, $G A$ index, $A B C$ index and harmonic index by using the linear programming methods.

\section{General results on VDB invariants}

Let $D$ be a digraph on $n \geq 2$ vertices without isolated vertices and $a_{i j}$ be the number of $\operatorname{arcs}$ of $D$ from vertices of out-degree $i$ to vertices of in-degree $j$. If $\varphi$ is symmetric, i.e. $\varphi_{i j}=\varphi_{j i}$ for all $1 \leq i<j \leq n-1$, then we can simplify the expression in (1) in the following

$$
I(D)=\frac{1}{2} \sum_{1 \leq i \leq j \leq n-1} p_{i j} \varphi_{i j}
$$

where $p_{i j}=a_{i j}+a_{j i}$ for $i \neq j$ and $1 \leq i, j \leq n-1$, and $p_{i i}=a_{i i}$ for all $i=1,2, \cdots, n-1$.

Note that $p_{i j}=p_{j i}$ for all $1 \leq i, j \leq n-1$, and

$$
\sum_{j=1}^{n-1} p_{i j}+p_{i i}=i n_{i}, \quad 1 \leq i \leq n-1
$$

where $n_{i}$ is the number of vertices of $D$ with out-degree $i$ or in-degree $i$. Also,

$$
n_{1}+n_{2}+\cdots+n_{n-1}=2 n-n_{0} .
$$

The digraphs with $n$ non-isolated vertices which satisfy the following conditions are of great interest to us

(i).

$$
\left\{\begin{array}{l}
p_{i j}=0 \text { for all }(i, j) \in\{(i, j) \mid 1 \leq i \leq j \leq n-1\}-\{(1, n-1)\} \\
n_{0}=0
\end{array}\right.
$$

i.e., a digraph with only $(1, n-1)$ - or $(n-1,1)$-arcs and the out-degree or in-degree of each vertex greater than 0 . The digraph obtained from the star on $n$ vertices by replacing each of its edges with a pair of symmetric arcs satisfies (5). The converse of this example does not hold since $D_{1}=(V, A)$ is also a digraph satisfied (5), where $V=\left\{v_{1}, v_{2}, \cdots, v_{n}\right\}$ and $A=\left\{v_{1} v_{2}, v_{2} v_{1}, v_{i} v_{1}, v_{2} v_{i} \mid 3 \leq i \leq n\right\}$.

(ii).

$$
\left\{\begin{array}{l}
p_{i j}=0 \text { for all }(i, j) \in\{(i, j) \mid 1 \leq i<j \leq n-1\} \\
n_{0}=n
\end{array}\right.
$$

i.e., the digraphs with only $(i, i)$-arcs $(1 \leq i \leq n-1)$ and the out-degree or in-degree of each vertex equal to $0 . \vec{K}_{2}$ satisfies (6), and $D_{2}=(V, A)$ is also a digraph satisfied (6), where $V=\left\{v_{1}, v_{2}, v_{3}, v_{4}\right\}$ and $A=\left\{v_{1} v_{3}, v_{1} v_{4}, v_{2} v_{3}, v_{2} v_{4}\right\}$. All digraphs in which each component is $\vec{K}_{2}$ or $D_{2}$ satisfy (6). 
(iii).

$$
\left\{\begin{array}{l}
p_{i j}=0 \text { for all }(i, j) \in\{(i, j) \mid 1 \leq i<j \leq n-1\} \\
n_{0}=0
\end{array}\right.
$$

i.e., the digraphs with only $(i, i)$-arcs $(1 \leq i \leq n-1)$ and the out-degree or in-degree of each vertex greater than 0 . The directed cycle $\vec{C}_{n}$ on $n$ vertices satisfies (7). All digraphs with $n$ non-isolated vertices in which each component is regular satisfy (7). The converse of this example does not hold since $D_{3}=(V, A)$ is also a digraph satisfied (7), where $V=\left\{v_{1}, v_{2}, v_{3}, v_{4}\right\}$ and $A=\left\{v_{1} v_{3}, v_{1} v_{4}, v_{2} v_{3}, v_{2} v_{4}, v_{3} v_{1}, v_{4} v_{2}\right\}$.

We try to find $\min (I(G))$ and $\max (I(G))$ under the constraints (3) and (4). The following results give the solutions of this problem for some VDB topological indices $I(D)$, i.e., determine the extremal values and the correspond extremal digraphs of $I(D)$ over all digraphs on $n$ vertices without isolated vertices.

Theorem 2.1. Let $D$ be a digraph on $n$ vertices without isolated vertices. Let

$$
L_{i j}=\frac{n-1}{n}\left(\frac{1}{i}+\frac{1}{j}\right) \varphi_{1, n-1} \quad \text { and } \quad S_{1}=\{(i, j) \mid 1 \leq i \leq j \leq n-1\}-\{(1, n-1)\} .
$$

Then

(i). If $\varphi_{i j}>L_{i j}$ for all $(i, j) \in S_{1}$, then $I(D) \geq \frac{n-1}{2} \varphi_{1, n-1}$ with equality if and only if $n_{0}=n$ and $p_{i j}=0$ for all $(i, j) \in S_{1}$, i.e., $D$ is the digraph $\vec{K}_{1, n-1}$ or $\vec{K}_{n-1,1}$, a star on $n$ vertices with its center of out-degree $n-1$ or 0 .

(ii). If $\varphi_{i j}<L_{i j}$ for all $(i, j) \in S_{1}$, then $I(D) \leq(n-1) \varphi_{1, n-1}$ with equality if and only if $n_{0}=0$ and $p_{i j}=0$ for all $(i, j) \in S_{1}$, i.e., D satisfies the conditions (5).

Proof. From (3), we have

$$
\begin{aligned}
n_{i}=\frac{1}{i}\left(\sum_{j=1}^{n-1} p_{i j}+p_{i i}\right), i=2,3, \cdots, n-2, & \\
n_{1}-p_{1, n-1} & =\sum_{j=1}^{n-2} p_{1 j}+p_{11}, \\
(n-1) n_{n-1}-p_{1, n-1} & =\sum_{j=2}^{n-1} p_{j, n-1}+p_{n-1, n-1} .
\end{aligned}
$$

By (4) and (8),

$$
n_{1}+n_{n-1}=2 n-n_{0}-\sum_{i=2}^{n-2} \frac{1}{i}\left(\sum_{j=1}^{n-1} p_{i j}+p_{i i}\right) .
$$

Multiplying (9) by $(n-1)$ and adding (10), we obtain

$$
(n-1)\left(n_{1}+n_{n-1}\right)-n p_{1, n-1}=(n-1) \sum_{j=1}^{n-2} p_{1 j}+(n-1) p_{11}+\sum_{j=2}^{n-1} p_{j, n-1}+p_{n-1, n-1},
$$

and by combining this equation with (11), we get

$$
\begin{aligned}
n p_{1, n-1} & =(n-1)\left(n_{1}+n_{n-1}\right)-(n-1)\left(\sum_{j=1}^{n-2} p_{1 j}+p_{11}\right)-\left(\sum_{j=2}^{n-1} p_{j, n-1}+p_{n-1, n-1}\right) \\
& =(n-1)\left[2 n-n_{0}-\sum_{i=2}^{n-2} \frac{1}{i}\left(\sum_{j=1}^{n-1} p_{i j}+p_{i i}\right)\right]-(n-1)\left(\sum_{j=1}^{n-2} p_{1 j}+p_{11}\right)-\left(\sum_{j=2}^{n-1} p_{j, n-1}+p_{n-1, n-1}\right) .
\end{aligned}
$$

Hence,

$$
\begin{aligned}
p_{1, n-1} & =2(n-1)-\frac{n-1}{n} n_{0}-\frac{n-1}{n} \sum_{i=2}^{n-2} \frac{1}{i}\left(\sum_{j=1}^{n-1} p_{i j}+p_{i i}\right)-\frac{n-1}{n}\left(\sum_{j=1}^{n-2} p_{1 j}+p_{11}\right)-\frac{1}{n} \sum_{j=2}^{n-1} p_{j, n-1}-\frac{1}{n} p_{n-1, n-1} \\
& =2(n-1)-\frac{n-1}{n} n_{0}-\frac{n-1}{n}\left[\sum_{i=1}^{n-1} \frac{1}{i}\left(\sum_{j=1}^{n-1} p_{i j}+p_{i i}\right)-\frac{n}{n-1} p_{1, n-1}\right] \\
& =2(n-1)-\frac{n-1}{n} n_{0}-\frac{n-1}{n}\left[\sum_{i=1}^{n-1} \frac{1}{i}\left(\sum_{j=1}^{n-1} p_{i j}+p_{i i}\right)\right]+p_{1, n-1} \\
& =2(n-1)-\frac{n-1}{n} n_{0}-\frac{n-1}{n}\left[\sum_{1 \leq i \leq j \leq n-1}\left(\frac{1}{i}+\frac{1}{j}\right) p_{i j}\right]+p_{1, n-1} \\
& =2(n-1)-\frac{n-1}{n} n_{0}-\frac{n-1}{n} \sum^{\prime}\left(\frac{1}{i}+\frac{1}{j}\right) p_{i j}
\end{aligned}
$$


where $\sum^{\prime}$ indicates summation over all $(i, j) \in S_{1}$. Substituting it into (2), we obtain

$$
\begin{aligned}
2 I(D) & =\varphi_{1, n-1} p_{1, n-1}+\sum^{\prime} \varphi_{i j} p_{i j} \\
& =\varphi_{1, n-1}\left[2(n-1)-\frac{n-1}{n} n_{0}-\frac{n-1}{n} \sum^{\prime}\left(\frac{1}{i}+\frac{1}{j}\right) p_{i j}\right]+\sum^{\prime} \varphi_{i j} p_{i j} \\
& =\left[2(n-1)-\frac{n-1}{n} n_{0}\right] \varphi_{1, n-1}+\sum^{\prime}\left[\varphi_{i j}-\frac{n-1}{n}\left(\frac{1}{i}+\frac{1}{j}\right) \varphi_{1, n-1}\right] p_{i j} .
\end{aligned}
$$

(i) If $\varphi_{i j}>L_{i j}=\frac{n-1}{n}\left(\frac{1}{i}+\frac{1}{j}\right) \varphi_{1, n-1}$ for all $(i, j) \in S_{1}$, then (12) shows that $I(D) \geq \frac{1}{2}\left[2(n-1)-\frac{n-1}{n} n_{0}\right] \varphi_{1, n-1}$. Moreover,

$$
I(D) \geq \frac{n-1}{2} \varphi_{1, n-1}
$$

since $n_{0} \leq n$, with equality if and only if $n_{0}=n$ and $p_{i j}=0$ for all $(i, j) \in S_{1}$, i.e., $D$ is the digraph $\vec{K}_{1, n-1}$ or $\vec{K}_{n-1,1}$.

(ii) If $\varphi_{i j}<L_{i j}$ for all $(i, j) \in S_{1}$, then (12) shows that $I(D) \leq \frac{1}{2}\left[2(n-1)-\frac{n-1}{n} n_{0}\right] \varphi_{1, n-1}$. Moreover, $I(D) \leq(n-1) \varphi_{1, n-1}$ since $n_{0} \geq 0$, with equality if and only if $n_{0}=0$ and $p_{i j}=0$ for all $(i, j) \in S_{1}$, i.e., $D$ is a digraph satisfied (5).

Theorem 2.2. Let $M_{i j}=\frac{n-1}{2}\left(\frac{1}{i}+\frac{1}{j}\right) \varphi_{n-1, n-1}$ and $S_{2}=\{(i, j) \mid 1 \leq i \leq j \leq n-1\}-\{(n-1, n-1)\}$. Then

(i). If $\varphi_{i j}>M_{i j}$ for all $(i, j) \in S_{2}$, then $I(D) \geq \frac{1}{4} n(n-1) \varphi_{n-1, n-1}$ with equality if and only if $n_{0}=n$ and $p_{i j}=0$ for all $(i, j) \in S_{2}$, i.e., $D=\vec{K}_{2}$.

(ii). If $\varphi_{i j}<M_{i j}$ for all $(i, j) \in S_{2}$, then $I(D) \leq \frac{1}{2} n(n-1) \varphi_{n-1, n-1}$ with equality if and only if $n_{0}=0$ and $p_{i j}=0$ for all $(i, j) \in S_{2}$, i.e., $D$ is the digraph obtained from $K_{n}$ by replacing each edge with a pair of symmetric arcs.

Proof. From (3) and (4), we obtain

$$
\begin{aligned}
n_{n-1} & =\left(2 n-n_{0}\right)-\sum_{i=1}^{n-2} \frac{1}{i}\left(\sum_{j=1}^{n-1} p_{i j}+p_{i i}\right) \\
& =\left(2 n-n_{0}\right)-\left(\sum_{j=1}^{n-1} \sum_{i=1}^{n-2} \frac{1}{i} p_{i j}+\sum_{i=1}^{n-2} \frac{1}{i} p_{i i}\right) \\
& =\left(2 n-n_{0}\right)-\sum_{1 \leq i \leq j \leq n-1}\left(\frac{1}{i}+\frac{1}{j}\right) p_{i j}+\frac{1}{n-1} \sum_{j=1}^{n-2} p_{j, n-1}+\frac{2}{n-1} p_{n-1, n-1} .
\end{aligned}
$$

By (3), it holds that

$$
\sum_{j=1}^{n-2} p_{n-1, j}+2 p_{n-1, n-1}=(n-1) n_{n-1}
$$

and

$$
\begin{aligned}
2 p_{n-1, n-1} & =(n-1) n_{n-1}-\sum_{j=1}^{n-2} p_{n-1, j} \\
& =(n-1)\left[\left(2 n-n_{0}\right)-\sum_{1 \leq i \leq j \leq n-1}\left(\frac{1}{i}+\frac{1}{j}\right) p_{i j}+\frac{1}{n-1} \sum_{j=1}^{n-2} p_{j, n-1}+\frac{2}{n-1} p_{n-1, n-1}\right]-\sum_{j=1}^{n-2} p_{n-1, j} \\
& =\left(2 n-n_{0}\right)(n-1)-(n-1) \sum^{\prime \prime}\left(\frac{1}{i}+\frac{1}{j}\right) p_{i j}
\end{aligned}
$$

where $\sum^{\prime \prime}$ indicates summation over all $(i, j) \in S_{2}$. By substituting it into (2), we obtain

$$
\begin{aligned}
2 I(D) & =\varphi_{n-1, n-1} p_{n-1, n-1}+\sum^{\prime \prime} \varphi_{i j} p_{i j} \\
& =\varphi_{n-1, n-1}\left[\frac{1}{2}\left(2 n-n_{0}\right)(n-1)-\frac{1}{2}(n-1) \sum^{\prime \prime}\left(\frac{1}{i}+\frac{1}{j}\right) p_{i j}\right]+\sum^{\prime \prime} \varphi_{i j} p_{i j} \\
& =\frac{1}{2}\left(2 n-n_{0}\right)(n-1) \varphi_{n-1, n-1}+\sum^{\prime \prime}\left[\varphi_{i j}-\frac{n-1}{2}\left(\frac{1}{i}+\frac{1}{j}\right) \varphi_{n-1, n-1}\right] p_{i j} .
\end{aligned}
$$

(i) If $\varphi_{i j}>M_{i j}$ for all $(i, j) \in S_{2}$, then (13) shows that $I(D) \geq \frac{1}{4}\left(2 n-n_{0}\right)(n-1) \varphi_{n-1, n-1}$. Moreover, $I(D) \geq \frac{1}{4} n(n-$ 1) $\varphi_{n-1, n-1}$ since $n_{0} \leq n$, with equality if and only if $n_{0}=n$ and $p_{i j}=0$ for all $(i, j) \in S_{2}$, i.e., $D$ is a digraph with only $(n-1, n-1)$-arcs and the out-degree or in-degree of each vertex equal to 0 . So, $D=\vec{K}_{2}$.

(ii) If $\varphi_{i j}<M_{i j}$ for all $(i, j) \in S_{2}$, then (13) shows that $I(D) \leq \frac{1}{4}\left(2 n-n_{0}\right)(n-1) \varphi_{n-1, n-1}$. Moreover, $I(D) \leq \frac{1}{2} n(n-$ 1) $\varphi_{n-1, n-1}$ since $n_{0} \geq 0$, with equality if and only if $n_{0}=0$ and $p_{i j}=0$ for all $(i, j) \in S_{2}$, i.e., $D$ is the digraph obtained from the complete graph $K_{n}$ by replacing each edge with a pair of symmetric arcs.

Theorem 2.3. Let $S_{3}=\{(i, j) \mid 1 \leq i \neq j \leq n-1\}$. Then

(i). If $\varphi_{i j}>M_{i j}$ for all $(i, j) \in S_{3}$, and $i \varphi_{i i}=(n-1) \varphi_{n-1, n-1}$ for $1 \leq i \leq n-2$, then $I(D) \geq \frac{1}{4} n(n-1) \varphi_{n-1, n-1}$ with equality if and only if $n_{0}=n$ and $p_{i j}=0$ for all $(i, j) \in S_{3}$, i.e., $D$ is a digraph satisfied (6). 
(ii). If $\varphi_{i j}<M_{i j}$ for all $(i, j) \in S_{3}$, and $i \varphi_{i i}=(n-1) \varphi_{n-1, n-1}$ for $1 \leq i \leq n-2$, then $I(D) \leq \frac{1}{2} n(n-1) \varphi_{n-1, n-1}$ with equality if and only if $n_{0}=0$ and $p_{i j}=0$ for all $(i, j) \in S_{3}$, i.e., $D$ is a digraph satisfied (7).

Proof. From (13), we have

$$
2 I(G)=\frac{1}{2}\left(2 n-n_{0}\right)(n-1) \varphi_{n-1, n-1}+\sum_{1 \leq i<j \leq n-1}\left[\varphi_{i j}-\frac{n-1}{2}\left(\frac{1}{i}+\frac{1}{j}\right) \varphi_{n-1, n-1}\right] p_{i j}+\sum_{i=1}^{n-2}\left[\varphi_{i i}-\frac{n-1}{i} \varphi_{n-1, n-1}\right] p_{i i} .
$$

(i) If $\varphi_{i j}>M_{i j}$ for all $(i, j) \in S_{3}$, and $i \varphi_{i i}=(n-1) \varphi_{n-1, n-1}$ for $1 \leq i \leq n-2$, then (14) shows that

$$
I(D) \geq \frac{1}{4}\left(2 n-n_{0}\right)(n-1) \varphi_{n-1, n-1} .
$$

Moreover, $I(D) \geq \frac{1}{4} n(n-1) \varphi_{n-1, n-1}$ since $n_{0} \leq n$, with equality if and only if $n_{0}=n$ and $p_{i j}=0$ for all $(i, j) \in S_{3}$, i.e., $D$ is the digraph satisfied (6).

(ii) If $\varphi_{i j}<M_{i j}$ for all $(i, j) \in S_{3}$, and $i \varphi_{i i}=(n-1) \varphi_{n-1, n-1}$ for $1 \leq i \leq n-2$, then (14) shows that

$$
I(D) \leq \frac{1}{4}\left(2 n-n_{0}\right)(n-1) \varphi_{n-1, n-1} .
$$

Moreover, $I(D) \leq \frac{1}{2} n(n-1) \varphi_{n-1, n-1}$ since $n_{0} \geq 0$, with equality if and only if $n_{0}=0$ and $p_{i j}=0$ for all $(i, j) \in S_{3}$, i.e., $D$ is a digraph satisfied (7).

Theorems 2.1-2.3 show that the results on digraphs are different from the results on graphs in [2].

\section{Applications}

In this section, we give some results on Randić index, Zagreb indices, sum-connectivity index, $G A$ index and $A B C$ index of digraphs by using Theorems 2.1-2.3.

\subsection{The general Randić index of digraphs}

Let $\varphi_{i j}=(i j)^{\alpha}$. Then $I(D)=R_{\alpha}(D)=\frac{1}{2} \sum_{1 \leq i \leq j \leq n-1} p_{i j}(i j)^{\alpha}$ is the general Randić index of a digraph $D$ with $n$ non-isolated vertices. In particular, $R_{\alpha}(D)$ is the Randic index, the second Zagreb index and the modified Zagreb index of a digraph for $\alpha=-\frac{1}{2}, \alpha=1$ and $\alpha=-1$, respectively.

(i) Let $-\frac{1}{2} \leq \alpha<+\infty$. Then $2 \alpha+1 \geq 0$.

Note that $i j \leq\left(\frac{i+j}{2}\right)^{2}$ and $i, j \leq n-1$, we have

$$
\begin{aligned}
(i j)^{\alpha+1} & \leq\left(\frac{i+j}{2}\right)^{2 \alpha+2}=\frac{1}{2^{2 \alpha+2}}(i+j)^{2 \alpha+1}(i+j) \\
& \leq \frac{1}{2^{2 \alpha+2}}[2(n-1)]^{2 \alpha+1}(i+j)=\frac{1}{2}(n-1)^{2 \alpha+1}(i+j),
\end{aligned}
$$

and $\varphi_{i j} \leq \frac{n-1}{2}\left(\frac{1}{i}+\frac{1}{j}\right) \varphi_{n-1, n-1}$ with equality if and only if (a) $i=j=n-1$ for $-\frac{1}{2}<\alpha<+\infty$, or (b) $i=j$ for $\alpha=-\frac{1}{2}$. By Theorems 2.2(ii) and 2.3(ii), we have

$$
R_{\alpha}(D) \leq \frac{1}{2} n(n-1) \varphi_{n-1, n-1}=\frac{1}{2} n(n-1)^{2 \alpha+1}
$$

with equality if and only if (a) $D$ is the digraph obtained from $K_{n}$ by replacing each edge with a pair of symmetric arcs for $-\frac{1}{2}<\alpha<+\infty$, or (b) $D$ is a digraph satisfied (7). So, (a) the digraph with the maximal general Randić index (including the second Zagreb index) for $-\frac{1}{2}<\alpha<+\infty$ is the digraph obtained from $K_{n}$ by replacing each edge with a pair of symmetric arcs; (b) the digraphs with the maximal Randić index are those satisfied (7), see Theorem 3.7 in [7].

Corollary 3.1. If $D \in \mathcal{D}_{n}$, then (a) $R_{\alpha}(D) \leq \frac{1}{2} n(n-1)^{2 \alpha+1}$ for $-\frac{1}{2}<\alpha<+\infty$ with equality if and only if $D$ is the digraph obtained from $K_{n}$ by replacing each edge with a pair of symmetric arcs; (b) (Theorem 3.7 in [7]) $R_{-\frac{1}{2}}(D) \leq \frac{n}{2}$ with equality if and only if D satisfies (7).

(ii) Let $-\infty<\alpha \leq-1$.

Because $i j \leq\left(\frac{i+j}{2}\right)^{2}$ and $\alpha \leq-1$, we have

$$
(i j)^{\alpha+1} \geq\left(\frac{i+j}{2}\right)^{2 \alpha+2}=\frac{1}{2^{2 \alpha+2}}(i+j)^{2 \alpha+1}(i+j)
$$




$$
\geq \frac{1}{2^{2 \alpha+2}}[2(n-1)]^{2 \alpha+1}(i+j)=\frac{1}{2}(n-1)^{2 \alpha+1}(i+j),
$$

and $\varphi_{i j} \geq \frac{n-1}{2}\left(\frac{1}{i}+\frac{1}{j}\right) \varphi_{n-1, n-1}$ with equality if and only if $i=j=n-1$. By Theorem 2.2(i), we have

$$
R_{\alpha}(D) \geq \frac{1}{4} n(n-1) \varphi_{n-1, n-1}=\frac{1}{4} n(n-1)^{2 \alpha+1}
$$

with equality if and only if $D=\vec{K}_{2}$. So, the digraph with the minimal general Randić index (including the modified Zagreb index) for $-\infty<\alpha \leq-1$ is $\vec{K}_{2}$.

Corollary 3.2. If $D \in \mathcal{D}_{n}$, then $R_{\alpha}(D) \leq \frac{1}{4} n(n-1)^{2 \alpha+1}$ for $-\infty<\alpha \leq-1$ with equality if and only if $D=\vec{K}_{2}$.

(iii) Let $-\frac{1}{2} \leq \alpha<0$.

In the following, we show that $\varphi_{i j}>\frac{n-1}{n}\left(\frac{1}{i}+\frac{1}{j}\right) \varphi_{1, n-1}$ for all $(i, j) \in\{(i, j) \mid 1 \leq i \leq j \leq n-1\}-\{(1, n-1)\}$. Let $g(x, y)=\frac{(x y)^{\alpha+1}}{x+y}$, where $1 \leq x \leq y \leq n-1$. Note that $\alpha x+y+\alpha y \geq(2 \alpha+1) x \geq 0, \frac{\partial g}{\partial x}=\frac{y(x y)^{\alpha}(\alpha x+y+\alpha y)}{(x+y)^{2}}=0$ and $\frac{\partial g}{\partial y}=\frac{x(x y)^{\alpha}(\alpha x+x+\alpha y)}{(x+y)^{2}}=0$ if and only if $\alpha=-\frac{1}{2}$ and $x=y$. So, the minimal point of $g(x, y)$ in the region $\{(x, y) \mid 1 \leq x \leq$ $y \leq n-1\}$ is on the boundary of this region, and the minimal value of $g(x, y)$ in the region $\{(x, y) \mid 1 \leq x \leq y \leq n-1\}$ is $\min \{g(1,1), g(1, n-1)\}=\min \left\{\frac{1}{2}, \frac{(n-1)^{\alpha+1}}{n}\right\}$. If $\alpha \in\left(-\frac{1}{2}, 0\right)$, then $\frac{(n-1)^{\alpha+1}}{n}<\frac{1}{2}$ for sufficiently large $n$; and if $\alpha=-\frac{1}{2}$, then $\frac{(n-1)^{\alpha+1}}{n}<\frac{1}{2}$ for $n \geq 3$. Hence, $g(i, j) \geq g(1, n-1)$, and

$$
(i j)^{\alpha} \geq \frac{(n-1)^{\alpha+1}}{n}\left(\frac{1}{i}+\frac{1}{j}\right) \text {, i.e. } \varphi_{i j} \geq \frac{n-1}{n}\left(\frac{1}{i}+\frac{1}{j}\right) \varphi_{1, n-1}
$$

with equality if and only if $(i, j)=(1, n-1)$. By Theorem 2.1(i), we have

$$
R_{\alpha}(D) \geq \frac{n-1}{2} \varphi_{1, n-1}=\frac{1}{2}(n-1)^{\alpha+1}
$$

with equality if and only if $D$ is the digraph $\vec{K}_{1, n-1}$ or $\vec{K}_{n-1,1}$ for sufficiently large $n$. So, the digraph with the minimal Randić index is $\vec{K}_{1, n-1}$ or $\vec{K}_{n-1,1}$ over $\mathcal{D}_{n}$ for $n \geq 3$; and the digraph with the minimal general Randić index for $\alpha \in\left(-\frac{1}{2}, 0\right)$ is also $\vec{K}_{1, n-1}$ or $\vec{K}_{n-1,1}$ over $\mathcal{D}_{n}$ for sufficiently large $n$.

Corollary 3.3. (a) (Theorem 3.11 in [7]) If $D \in \mathcal{D}_{n}, n \geq 3$, then $R_{-\frac{1}{2}}(D) \geq \frac{1}{2} \sqrt{n-1}$ with equality if and only if $D=\vec{K}_{1, n-1}$ or $D=\vec{K}_{n-1,1}$;

(b) Let $-\frac{1}{2} \leq \alpha<0$. If $D \in \mathcal{D}_{n}$, then $R_{\alpha}(D) \geq \frac{1}{2}(n-1)^{\alpha+1}$ for sufficiently large $n$, with equality if and only if $D=\vec{K}_{1, n-1}$ or $D=\vec{K}_{n-1,1}$.

\subsection{The general sum-connectivity index of digraphs}

Let $\varphi_{i j}=(i+j)^{\alpha}$. Then $I(D)=\chi_{\alpha}(D)=\frac{1}{2} \sum_{1<i<j<n-1} p_{i j}(i+j)^{\alpha}$ is the general sum-connectivity index of a digraph $D$, and $\chi_{\alpha}(D)$ is the sum-connectivity index and the first Zagreb index of $D$ for $\alpha=-\frac{1}{2}$ and $\alpha=1$, respectively.

(i) Let $-1 \leq \alpha<+\infty$.

Because $1 \leq i \leq j \leq n-1$ and $\alpha+1 \geq 0$,

$$
\begin{aligned}
i j & \leq\left(\frac{i+j}{2}\right)^{2}=\left(\frac{i+j}{2}\right)^{1-\alpha}\left(\frac{i+j}{2}\right)^{1+\alpha} \\
& \leq\left(\frac{i+j}{2}\right)^{1-\alpha}(n-1)^{1+\alpha},
\end{aligned}
$$

and $\varphi_{i j}=(i+j)^{\alpha} \leq \frac{n-1}{2}\left(\frac{1}{i}+\frac{1}{j}\right)[2(n-1)]^{\alpha}=\frac{n-1}{2}\left(\frac{1}{i}+\frac{1}{j}\right) \varphi_{n-1, n-1}$ with equality if and only if (a) $i=j=n-1$ for $-1<\alpha<+\infty$, or (b) $i=j$ for $\alpha=-1$. By Theorems 2.2(ii) and 2.3(ii), we have

$$
\chi_{\alpha}(D) \leq \frac{1}{2} n(n-1) \varphi_{n-1, n-1}=2^{\alpha-1} n(n-1)^{\alpha+1}
$$

with equality if and only if (a) $D$ is the digraph obtained from the complete graph $K_{n}$ by replacing each edge with a pair of symmetric arcs, or (b) $D$ satisfies (7). Especially, this shows that the graph with the maximal sum-connectivity index, or the maximal first Zagreb index is $K_{n}$ among all graphs of order $n$.

Corollary 3.4. If $D \in \mathcal{D}_{n}$, then (a) $\chi_{\alpha}(D) \leq 2^{\alpha-1} n(n-1)^{\alpha+1}$ for $-\frac{1}{2}<\alpha<+\infty$ with equality if and only if $D$ is the digraph obtained from $K_{n}$ by replacing each edge with a pair of symmetric arcs; (b) $\chi_{-1}(D) \leq \frac{n}{4}$ with equality if and only if $D$ satisfies (7). 
(ii) Let $-1 \leq \alpha<0$.

We consider the function $g(x, y)=(x y)(x+y)^{\alpha-1}$, where $1 \leq x \leq y \leq n-1$. It is easy to know that the minimal value of $g(x, y)=(x y)(x+y)^{\alpha-1}$ in the region $\{(x, y) \mid 1 \leq x \leq y \leq n-1\}$ is $\min \{g(1,1), g(1, n-1)\}=\min \left\{2^{\alpha-1},(n-1) n^{\alpha-1}\right\}$. If $\alpha \in\left(-\frac{1}{2}, 0\right)$, then $(n-1) n^{\alpha-1}<2^{\alpha-1}$ for sufficiently large $n$; and if $\alpha \in\left[-1,-\frac{1}{2}\right]$, then $(n-1) n^{\alpha-1}<2^{\alpha-1}$ for $n \geq 6$. Hence, $g(i, j) \geq g(1, n-1)$, and

$$
(i+j)^{\alpha} \geq(n-1) n^{\alpha-1}\left(\frac{1}{i}+\frac{1}{j}\right), \text { i.e., } \quad \varphi_{i j} \geq \frac{n-1}{n}\left(\frac{1}{i}+\frac{1}{j}\right) \varphi_{1, n-1}
$$

with equality if and only if $(i, j)=(1, n-1)$. By Theorem 2.1(i), we have

$$
\chi_{\alpha}(D) \geq \frac{1}{2}(n-1) \varphi_{1, n-1}=\frac{1}{2}(n-1) n^{\alpha}
$$

with equality if and only if $D$ is $\vec{K}_{1, n-1}$ or $\vec{K}_{n-1,1}$ for $\alpha \in[-1,0)$ and sufficiently large $n$, or for $\alpha \in\left[-1,-\frac{1}{2}\right]$ and $n \geq 6$. So that the graph with the minimal general sum-connectivity index for $\alpha \in\left[-1,-\frac{1}{2}\right]$ is $\vec{K}_{1, n-1}$ or $\vec{K}_{n-1,1}$ over $\mathcal{D}_{n}$; and the digraph with the minimal general sum-connectivity index for $\alpha \in[-1,0)$ is also $\vec{K}_{1, n-1}$ or $\vec{K}_{n-1,1}$ over $\mathcal{D}_{n}$ when $n$ is sufficiently large.

Corollary 3.5. Let $D \in \mathcal{D}_{n}$. If $\alpha \in\left[-1,-\frac{1}{2}\right]$ and $n \geq 6$, or $\alpha \in[-1,0)$ and $n$ is sufficiently large, then $\chi_{\alpha}(D) \geq \frac{1}{2}(n-1) n^{\alpha}$ with equality if and only if $D$ is $\vec{K}_{1, n-1}$ or $\vec{K}_{n-1,1}$.

\subsection{The geometric-arithmetic index of digraphs}

Let $\varphi_{i j}=\frac{\sqrt{i j}}{\frac{1}{2}(i+j)}$. Then $I(D)=G A(D)=\frac{1}{2} \sum_{1 \leq i \leq j \leq n-1} p_{i j} \frac{\sqrt{i j}}{\frac{1}{2}(i+j)}$ is the first geometric-arithmetic index $G A$ of a digraph $D$.

(i) Note that $\varphi_{n-1, n-1}=1$ and $(i j)^{\frac{3}{2}} \leq\left(\frac{i+j}{2}\right)^{3}=\frac{i+j}{8}(i+j)^{2} \leq \frac{n-1}{4}(i+j)^{2}$, i.e. $\frac{\sqrt{i j}}{\frac{1}{2}(i+j)} \leq \frac{n-1}{2}\left(\frac{1}{i}+\frac{1}{j}\right)$, we have $\varphi_{i j} \leq$ $\frac{n-1}{2}\left(\frac{1}{i}+\frac{1}{j}\right) \varphi_{n-1, n-1}$ with equality if and only if $i=j=n-1$. By Theorem 2.2(ii),

$$
G A(D) \leq \frac{1}{2} n(n-1) \varphi_{n-1, n-1}=\frac{1}{2} n(n-1)
$$

with equality if and only if $D$ is the digraph obtained from $K_{n}$ by replacing each edge with a pair of symmetric arcs.

(ii) It is easy to know that the minimal value of $g(x, y)=\frac{(x y)^{\frac{3}{2}}}{(x+y)^{2}}$ in the region $\{(x, y) \mid 1 \leq x \leq y \leq n-1\}$ is $g(1, n-1)=$ $\frac{(n-1)^{\frac{3}{2}}}{n^{2}}, g(i, j) \geq g(1, n-1)$, i.e. $\frac{(i j)^{\frac{3}{2}}}{(i+j)^{2}} \geq \frac{(n-1)^{\frac{3}{2}}}{n^{2}}$. Hence,

$$
\frac{\sqrt{i j}}{\frac{1}{2}(i+j)} \geq \frac{n-1}{n}\left(\frac{1}{i}+\frac{1}{j}\right) \frac{\sqrt{n-1}}{\frac{1}{2} n} \quad \text { i.e., } \quad \varphi_{i j} \geq \frac{n-1}{n}\left(\frac{1}{i}+\frac{1}{j}\right) \varphi_{1, n-1}
$$

with equality if and only if $(i, j)=(1, n-1)$. By Theorem 2.1(i), we have

$$
G A(D) \geq \frac{1}{2}(n-1) \varphi_{1, n-1}=\frac{(n-1)^{\frac{3}{2}}}{n}
$$

with equality if and only if $D$ is $\vec{K}_{1, n-1}$ or $\vec{K}_{n-1,1}$.

So, we obtain the digraphs with the maximal and the minimal geometric-arithmetic index $G A$ over $\mathcal{D}_{n}$.

Corollary 3.6. If $D \in \mathcal{D}_{n}$, then $G A(D) \leq \frac{1}{2} n(n-1)$ with equality if and only if $D$ is the digraph obtained from $K_{n}$ by replacing each edge with a pair of symmetric arcs; $G A(D) \geq \frac{(n-1)^{\frac{3}{2}}}{n}$ with equality if and only if $D$ is $\vec{K}_{1, n-1}$ or $\vec{K}_{n-1,1}$.

\subsection{The atom-bond connectivity index of digraphs}

Let $\varphi_{i j}=\sqrt{\frac{i+j-2}{i j}}$, then $I(D)=A B C(D)=\frac{1}{2} \sum_{1 \leq i \leq j \leq n-1} p_{i j} \sqrt{\frac{i+j-2}{i j}}$ is the ABC index of a digraph $D$. Since $1 \leq i \leq j \leq$ $n-1$,

$$
\frac{i+j-2}{i j} \leq \frac{2(n-2)}{i j} \leq \frac{2(n-2)}{(i j)^{2}}\left(\frac{i+j}{2}\right)^{2} \leq \frac{n-2}{2}\left(\frac{i+j}{i j}\right)^{2},
$$

and $\sqrt{\frac{i+j-2}{i j}} \leq \sqrt{\frac{n-2}{2}}\left(\frac{i+j}{i j}\right)$, i.e., $\varphi_{i j} \leq \frac{n-1}{2}\left(\frac{1}{i}+\frac{1}{j}\right) \varphi_{n-1, n-1}$ with equality if and only if $i=j=n-1$. By Theorem 2.2(ii), we have

$$
A B C(D) \leq \frac{1}{2} n(n-1) \varphi_{n-1, n-1}=\frac{1}{2} n \sqrt{2 n-4}
$$

with equality if and only if $D$ is the digraph obtained from $K_{n}$ by replacing each edge with a pair of symmetric arcs.

This shows that the digraphs with the maximal $\mathrm{ABC}$ index over $\mathcal{D}_{n}$ is the digraph obtained from $K_{n}$ by replacing each edge with a pair of symmetric arcs.

Corollary 3.7. If $D \in \mathcal{D}_{n}$, then $A B C(D) \leq \frac{1}{2} n \sqrt{2 n-4}$ with equality if and only if $D$ is the digraph obtained from $K_{n}$ by replacing each edge with a pair of symmetric arcs. 


\subsection{The harmonic index of digraphs}

Let $\varphi_{i j}=\frac{2}{i+j}$. Then $I(D)=h(D)=\frac{1}{2} \sum_{1 \leq i \leq j \leq n-1} p_{i j} \frac{2}{i+j}$ is the harmonic index of a digraph $D$.

(i) Note that

$$
\varphi_{i j}=\frac{2}{i+j} \leq \frac{i+j}{2 i j}=\frac{n-1}{2}\left(\frac{1}{i}+\frac{1}{j}\right) \varphi_{n-1, n-1}
$$

with equality if and only if $i=j$, and $i \varphi_{i i}=1=(n-1) \varphi_{n-1, n-1}$, from Theorem 2.3(ii), we have

$$
h(D) \leq \frac{1}{2} n(n-1) \varphi_{n-1, n-1}=\frac{n}{2}
$$

with equality if and only if $D$ is a digraph satisfied (7).

(ii) Also, the minimal value of $g(x, y)=\frac{(x y)}{(x+y)^{2}}$ in the region $\{(x, y) \mid 1 \leq x \leq y \leq n-1\}$ is $g(1, n-1)=\frac{n-1}{n^{2}}$, we have $g(i, j)=\frac{i j}{(i+j)^{2}} \geq \frac{n-1}{n^{2}}=g(1, n-1)$ and

$$
\varphi_{i j}=\frac{2}{i+j} \geq \frac{n-1}{n}\left(\frac{1}{i}+\frac{1}{j}\right) \frac{2}{n}=\frac{n-1}{n}\left(\frac{1}{i}+\frac{1}{j}\right) \varphi_{1, n-1}
$$

with equality if and only if $(i, j)=(1, n-1)$. By Theorem 2.1(i), we have

$$
h(D) \geq \frac{1}{2}(n-1) \varphi_{1, n-1}=\frac{n-1}{n}
$$

with equality if and only if $D$ is $\vec{K}_{1, n-1}$ or $\vec{K}_{n-1,1}$.

So, we obtain the digraphs with the minimal and maximal harmonic index over $\mathcal{D}_{n}$.

Corollary 3.8. If $D \in \mathcal{D}_{n}$, then $h(D) \leq \frac{n}{2}$ with equality if and only if $D$ is a digraph satisfied $(7) ; h(D) \geq \frac{n-1}{n}$ with equality if and only if $D$ is $\vec{K}_{1, n-1}$ or $\vec{K}_{n-1,1}$.

\section{Acknowledgment}

This work is supported by the Hunan Provincial Natural Science Foundation of China (through grant no. 2020JJ4423), the Department of Education of Hunan Province (through grant no. 19A318) and the National Natural Science Foundation of China (through grant no. 11971164).

\section{References}

[1] B. Bollobás, P. Erdős, Graphs of extremal weights, Ars Combin. 50 (1981) 225-233.

[2] H. Deng, G. Huang, X. Jiang, A unified linear-programming modeling of some topological indices, J. Comb. Optim. 30 (2015) $826-837$.

[3] E. Estrada, L. Torres, L. Rodríguez, I. Gutman, An atom-bond connectivity index: Modelling the enthalpy of formation of alkanes, Indian J. Chem. A 37 (1998) 849-855.

[4] S. Fajtlowicz, On conjectures of Graffiti-II, Congr. Numer. 60 (1987) 187-197.

[5] I. Gutman, K. C. Das, The first Zagreb index 30 years after, MATCH Commun. Math. Comput. Chem. 50 (2004) 83-92.

[6] I. Gutman, B. Furtual (Eds.), Recent Results in the Theory of Randic Index, Univ. Kragujevac, Kragujevac, 2008.

[7] J. Monsalve, J. Rada, Vertex-degree based topological indices of digraphs, Discrete Appl. Math. 295 (2021) 13-24.

[8] S. Nikolić, G. Kovačcević, A. Miličcević, N. Trinajstić, The Zagreb indices 30 years after, Croat. Chem. Acta 76 (1972) 113-124.

[9] M. Randić, On characterization of molecular branching, J. Amer. Chem. Soc. 97 (1975) 6609-6615.

[10] D. Vukičević, B. Furtula, Topological index based on the ratios of geometrical and arithmetical means of end-vertex degrees of edges, J. Math. Chem. 6 (2009) 1369-1376.

[11] B. Zhou, N. Trinajstić, On a novel connectivity index, J. Math. Chem. 46 (2009) 1252-1270.

[12] B. Zhou, N. Trinajstić, On general sum-connectivity index, J. Math. Chem. 47 (2010) 210-218. 